\title{
$\sqrt{6}$ \\ AGUAYTERRIORIO
}

\section{El aguador y la infraestructura hidráulica en la ciudad de Guadalajara, México}

\section{The Water Carrier and the Hydraulic Infrastructure in Guadalajara, Mexico}

J osé J uan Pablo Rojas-Ramírez

Universidad de Guadalajara. Tonalá, México. jpablo.rojas@cutonala.udg.mx

\begin{abstract}
Resumen - Se describe la dinámica del aguador y su paulatina transformación en empleado repartidor de agua durante el proceso de implementación de la infraestructura hidráulica en la ciudad de Guadalajara. El desarrollo económico de la ciudad, su expansión territorial y el aumento demográfico frente a la escasez de agua obligaron a las autoridades a desarrollar proyectos hidráulicos con el fin de garantizar el abastecimiento de agua en diferentes periodos.

Abstract - This paper offers a description, grounded on a critical assessment of the past, about the transformation of traditional water carriers to the modernday water delivery employees. In doing so the technical procedures used to supply water to the Mexican city of Guadalajara are also examined. These mechanisms contributed to the transformation attending the expansion of the different areas of production, which prompted the city officials to implement several projects to guarantee the volume of water needed. This activity modified the various methods that were being used, which expanded from surface exploration to analyses of the feasibility of exploiting water aquifers to meet the urgent needs of a growing population and industry, all of which had a detrimental impact on the trade of the water carriers.
\end{abstract}

Palabras clave: aguador, proyectos hidráulicos, Guadalajara, México
Keywords: water-carriers, hydraulic projects, Guadalajara city, Mexico

Información Artículo: Recibido: 7 febrero 2016

Revisado: 30 enero 2017

Aceptado: 26 mayo 2016

(c) Universidad de J aén / Seminario Permanente Agua, Territorio y Medio Ambiente (CSIC) 


\section{INTRODUCCIÓN}

Se describe la dinámica del aguador frente a la introducción de infraestructura hidráulica, cada vez más tecnificada, en la ciudad de Guadalajara y se busca la correlación del desarrollo hidráulico con la transformación del oficio del prestador de servicios mencionado. Para tal efecto se realiza una retrospección sobre el manejo y distribución del agua en la ciudad, desde sus inicios en el valle de Atemajac.

En la cuestión de la retrospección crítica histórica se establece un diálogo entre lo presente y pasado, no con el fin de rescatar el pasado o un fragmento del mismo: lo que interesa es cómo manifestaron las gentes su humanidad y las relaciones con el entorno.

Se utilizó el método analítico cuya premisa consiste en fragmentar la realidad en las partes componentes del contexto social del aguador para explicarlo. Se tomaron como referencia los recursos bibliográficos existentes que aluden a la dinámica del aguador en Guadalajara y se ofrece una explicación sobre su devenir durante las fases de construcción de obras hidráulicas.

De manera colateral se enuncian los cambios de pauta cultural en cuanto al uso del agua: del comunitario en fuentes públicas y ríos a uno segmentado y hasta privatizado a través de la introducción de tubería a cada casa tapatíal de acuerdo al modelo higienista anglosajón cuyas pautas se adoptaron como regla social en los hábitos de higiene.

En la medida que la ciudad crecía y se consolidaba económicamente surgió la necesidad de requerir agentes que facilitaran el traslado de agua potable a las viviendas o comercios procedente de fuentes naturales cada vez más lejanas. Por ello se recurrió, en un principio, a la implementación del oficio del aguador, y posteriormente a la construcción de obras hidráulicas.

El aguador era un individuo que trasladaba agua de fuentes naturales a usuarios que requerían de su servicio. En la dinámica mexicana se observa que este prestador de servicios, al que se le consideró miembro del gremio de los arrieros, poseía rasgos distintivos al resto de los otros agremiados. A diferencia de los mensaj eros y transportistas, el aguador evidencia rasgos artesanales en su vestimenta y sus instrumentos de trabaj o: los cántaros decorados y las carretillas adaptadas para transportar agua; además de cumplir criterios de limpieza exigidos por las autoridades.

El gremio, en especial el segmento de los aguadores, estaba regulado por restricciones administrativas en cuanto a la organización de grupos para la distribución del trabajo y la delimitación territorial en la que ejercían el oficio. De acuerdo a las fuentes consultadas también experimentaron restricciones sociales de segmentación social, ya que en sus inicios fue ejercido por personas de estratos socioeconómicos bajos. Al aguador se le describe como un actor indispensable, aunque poco valorado, en los escritos de Sartorius ${ }^{2}$ y se define el oficio como propio de indios 0 campesinos.

1 De acuerdo a la Real Academia Española, tapatío o tapatía es el gentilicio que se usa para nombrar a una persona u obj eto relativo o que procede de Guadalajara, J alisco.

2 Sartorius, 1991.

\section{EL OFICIO de AGUAdOR Y EL MANEJ O DEL AGUA EN EL PERÍOdO COLONIAL EN LA CIUDAD DE GUADALAJ ARA}

El argumento de la escasez de agua en la ciudad de Guadalajara (México) tiene un antecedente desde su fundación en el Valle de Atemajac, en cuya época se manifestó la insuficiencia del recurso para atender las necesidades de la población tapatía3. Habría que preguntarse si la escasez en 1542, año en que se funda la ciudad, responde a los mismos criterios de una demanda creciente y diversificada en múltiples actividades, comerciales, industriales y sociales, como en periodos posteriores.

En términos reduccionistas, en un principio la solución para la escasez hídrica en la ciudad dependía de dos factores, uno relacionado al estudio de fuentes naturales propicias para el abastecimiento y otro que versa sobre la introducción de un oficio tradicional árabe-español: el aguador, que proveía del recurso hídrico en vasijas elaboradas en las localidades de Tlaquepaque y Tonalá, que se caracterizaron por su vocación alfarera.

En un principio el aguador era poco demandado por la población, ya que solo se recurría a él si los recursos económicos de los habitantes les permitían pagar un servicio extra como la dotación de agua limpia. Además, las necesidades de agua en los inicios de la ciudad en el valle de Atemajac eran solventadas a través de la sustracción de un riachuelo cercano. Dicha situación cambió una vez que la ciudad se consolidó económicamente y se incrementó la necesidad de agua salubre, dadas las condiciones de contaminación de riachuelos cercanos como el de San J uan de Dios.

"Incluso desde tiempos muy tempranos existía la costumbre de ad-
quirir el agua para el consumo a los aguadores que la Ilevaban de
lugares un poco más lejanos y más higiénicos. Un testimonio de
[obispo Alonso de la Mota y Escobar] a principios de siglo XVII expli-
ca que 'el agua que se / b/ ebe comúnmente en esta ciudad es de
algunas fuentes cercanas que tienen manantiales de agua razona-
ble': los regalados / b/ eben de otros ríos y fuentes mejores que son
distantes dos o tres leguas" 4 .

Conforme la ciudad de Guadalajara crecía, se incrementó la necesidad de recurrir a las autoridades para que estas socorrieran con ayuda técnica en el estudio de fuentes naturales viables que pudieran proveer de más cantidad de agua y acercarla a la ciudad a través de tecnología hidráulica ya que los aguadores no daban abasto a los pobladores.

A esta situación es preciso sumar que, mientras la población se percataba de la existencia de agua salubre procedente de ríos y manantiales, cada vez más alejados a la ciudad, el proceso burocrático para implementar obras se prolongaba también en el tiempo. Esto debido a las distancias existentes entre las autoridades competentes en cada ámbito de gobierno durante la época colonial en Nueva España.

Dadas las condiciones de precariedad hídrica en Guadalajara y lo dilatado del proceso burocrático se puede inferir que la tensión social surgía y con ello el conflicto, al cual se daba respuesta

\footnotetext{
3 Pérez Peña y Torres González, 2001, 5.

4 Ávila y González, 2012, 13.
} 
una vez que se enviaba la ayuda técnica y se concretaba la abundancia de agua, aunque por corto tiempo.

Con el paso del tiempo, la dinámica de "encontrar" fuentes de abastecimiento y aprobar su apropiación, fue adquiriendo mayor complejidad. Debido a la expansión territorial de la ciudad y al incremento de las dinámicas productivas, el manejo del agua requirió que se incorporaran criterios técnicos cada vez más sofisticados que involucraron la ejecución de proyectos con mayor tecnificación para garantizar a largo plazo la existencia del recurso.

La incorporación de diversos criterios técnicos mediante influjos de maquinaria hizo más eficiente el proceso tanto en la búsqueda de agua como en su apropiación al lograrse certeza en la existencia del recurso a corto y largo plazo para las crecientes poblaciones urbanas que se incorporaban a la ciudad 5 . A pesar de la adopción de criterios técnicos la función del aguador siguió vigente, aunque en otros términos.

Las dinámicas de abasto de agua presentan diferentes procedimientos en cada periodo. Los tiempos de estiaje incidían en el desarrollo de las actividades de los tapatíos; por ejemplo la situación de escasez y abasto del recurso para las actividades de los habitantes de Guadalajara en 1542 no se resolvió de la misma manera que en el año 1731. En el primer periodo la decisión incumbía solo a los habitantes bajo la venia institucional del virreinato y del imperio, cuando el estiaje amenazaba se llevaban a cabo estudios, un tanto empíricos, sobre la factibilidad de trasladar agua de fuentes más lejanas al centro de población $n^{6}$. La principal captación de agua se realizaba de los manantiales del suroriente de la ciudad y del riachuelo San J uan de Dios en el centro de la ciudad, que en menos de doscientos años se tornó insuficiente para el incremento de población y la dinámica económica.

En 1731 la situación referida a la captación y distribución de agua tuvo un carácter más institucional en los estudios de factibilidad realizados. Dicha circunstancia se logró, por un lado, por el apaciguamiento de los grupos indígenas y, por otro, por la estabilidad lograda por el imperio español; por consiguiente, se requería de la autorización de la jerarquía mayor y de la ayuda económica de mecenas para realizar estudios más sistemáticos y construir obras hidráulicas ${ }^{7}$.

La captación de agua en 1542 se atribuyó a los colonizadores, que guiados por los preceptos del rey y de los criterios de urbanización, buscaron lugares idóneos para el desarrollo de poblaciones. Al asentarse en el Valle de Atemajac la primera apreciación fue la de ser un valle fértil y con abundante agua; sin embargo, el estiaje producido por el cambio de estación cambió dicha apreciación.

Durante ese periodo, los habitantes se abastecieron de agua de los nacimientos naturales y del riachuelo San J uan de Dios, sin tener en cuenta la multiplicación futura de habitantes. Cuando esto aconteció se recurrió a la realización de estudios que garantizaran el traslado efectivo de agua procedente de diferentes

\footnotetext{
5 Pérez Peña y Torres González, 2001. Torres Rodríguez, 2013a, 2013b.

6 Torres Rodríguez, 2013a.

7 Rojas Ramírez, 2013.
}

fuentes naturales: "Desde 1597 se intentó por primera vez traer el agua de Los Colomos, como lo señala López Cotilla en 1842. Sin embargo, no hubo resultados adecuados" ${ }^{\prime 8}$.

Ante las imposibilidades observadas, se introdujo un oficio añejo en la Península Ibérica -el aguador-como proveedor de agua que acarreaba de un lugar a otro el recurso hídrico. A este personaje se le consideraba persona de confianza?.

El oficio del aguador y las funciones colaterales que se le asignaron contribuyeron a construir una versión "polifacética" de este prestador de servicios en la ciudad: por un lado distribuía agua de diferentes puntos periféricos, en momentos de incendio era auxiliar en la extinción del fuego, en frecuentes ocasiones auxiliaba en la comunicación y difusión de mensajes entre la población.

En la mayoría de los casos ahorraba la fatiga de las clases acaudaladas, asentadas al poniente de la ciudad, al transportar agua procedente de las fuentes públicas cuando estas se diversificaron ${ }^{10}$ y cuando en los pozos y norias construidos al interior de cada casa escaseaba el agua.

Para 1731 el manejo del agua y el desarrollo de la obra hidráulica se realizaron mediante estudios técnicos complejos, con ayuda económica de las autoridades coloniales. Para efectuar obras de captación de agua se requería de la autorización de la jerarquía estamental mayor y de su anuencia económica para implementar dichas obras. Por ejemplo, el superintendente de aguas, oidor de la audiencia y marqués de Altamira, solicitó al fraile franciscano Pedro Buzeta que estudiara la posibilidad de introducir agua en la ciudad.

Tras dos años de estudios basados en la comprensión del sistema hídrico y topográfico del entorno, el religioso concluyó que, de todas las aguas estudiadas, las de mayor viabilidad eran las del río Tototlán, el riachuelo San J uan de Dios y las aguas provenientes de veneros al oriente de la ciudad -como las de Agua Azul, el río Zapopan y río Blanco, estos dos últimos al poniente de la ciudad-.

Sin embargo, solo una de estas podría garantizar el abasto por cierto tiempo, teniendo en cuenta el conocimiento adquirido por casi doscientos años y la capacidad técnica para determinar la utilización eficiente de fuentes subterráneas.

Entonces, las aguas subterráneas del poniente de la ciudad fueron utilizadas para abastecer a la población mediante la construcción de tres bóvedas que conducían el recurso hacia diferentes direcciones en las que desembocaban tubos que abastecían fuentes públicas. La obra fue tan ingeniosa que causó la admiración y la detracción durante mucho tiempo. Incluso en la época independiente abundaron comentarios, como el de Manuel López Cotilla:

"...es una obra ingeniosa que fue dirigida por fray Pedro Buzeta... Consiste en tres líneas de pozos que comenzando en un punto, se abren en dos ángulos muy prolongados que se extienden por el de-

\footnotetext{
8 Torres Rodríguez, 2013a.

9 Sartorius, 1991.

10 Arana Cervantes, 1980, 28-29.
} 
clive de una loma árida y sin ninguna agua en superficie, pero que la tiene a una profundidad dominante todavía respecto a la ciudad. Estos pozos se comunican por cañones subterráneos que facilitan el curso del agua... Como el agua viene de pozos, su abundancia 0 escasez depende de las que tienen las lluvias, y regularmente disminuye en tiempo que es más necesaria" ${ }^{11}$.

Las galerías construidas por idea de Buzeta, funcionaron casi cien años, el éxito se evidenció con la accesibilidad del recurso. Esto propició que la distribución del agua fuera más eficiente en fuentes públicas. Los aguadores reestructuraron su organización, ya que surgió la necesidad de fragmentar el territorio y asignar a cada sector un grupo con el fin de administrar el llenado de los cántaros desde las fuentes y trasladarlos hasta los domicilios particulares sin que existiera competencia entre cada uno de ellos.

Además, se dinamizó y facilitó la actividad del aguador, ya que se redujo el tiempo de traslado de los nacimientos naturales en torno a los municipios contiguos: Zapopan, Tlaquepaque y Tonalá, cuyos afluentes se encuentran entre los 15 y 20 kilómetros, que se recorrían entre el centro de la ciudad y los afluentes naturales. El trayecto se redujo de 4 a 8 kilómetros en promedio.

En cierta medida, la elaboración del proyecto de fray Pedro Buzeta sería el antecedente de las políticas hidráulicas modernas para la ciudad, con la salvedad de las complejidades sociales, políticas y económicas que enmarcan el diseño, implementación y evaluación de la obra hidráulica moderna, ya que en este proceso se considera el aforo presente y en teoría debería garantizarse la existencia futura bajo un cuidado óptimo de la fuente.

"... se siguieron analizando... las aguas de Los Colomos (1777) por el oidor Zagarzurrieta; del Astillero, del Alamo, las fuentes de Zapote y Buelles, presa antigua, la de la Huerta o Camichin (1787); Mexicalzingo (1801)... río Grande-río Santiago-San Ramón, Los Colomos (1830)..." 12

Ante lo innovador de las apreciaciones de Buzeta y los múltiples proyectos existentes desde el siglo XVIII, la incapacidad económica y la poca capacidad de retención de los suelos porosos menguaban el abasto de agua a la población ${ }^{13}$. Por consiguiente, el aguador siguió siendo la opción para proveer de agua al consumo humano, a pesar de los proyectos realizados por el ingeniero Castaños.

\section{EL OFICIO de AGUAdOR Y LA INTRODUCCIÓN DE TECNOLOGía HIDRÁULICA EN LA MODERNIDAD: ENTRE DOS SIGLOS DE TRANSICIÓN}

Durante el siglo XIX y a principios del XX, los criterios para ej ecutar obras destinadas a combatir la escasez de agua se basaron principalmente en el uso comunal del recurso con un progresivo cambio hacia la introducción de un sistema de distribución de agua a cada vivienda, así como en la disposición de aguas residuales a una red de alcantarillado.

Mientras tanto, el aguador surtía de agua potable a la población y las autoridades locales ideaban nuevas obras de tecno-

\footnotetext{
11 López Cotilla, 1983

12 Torres Rodríguez, 2013a.

13 Ibidem.
}

logía hidráulica. Respecto al perfil de estos prestadores de servicio cabe destacar que en la mayoría de los casos eran indígenas, campesinos o personas empobrecidas, según datan los documentos oficiales y fotografías localizadas en el Archivo municipal de Guadalajara. Todo aquel que aspiraba a ejercer el oficio poseía carretilla, cántaros de barro y, en algunos casos, mulas o burros que aprovechaban en conjunto para comercializar el agua.

En este periodo se transitó de la dinámica de abastecimiento a través de fuentes públicas a la introducción de tubería a cada casa con el fin de tener un abastecimiento eficaz. En Guadalajara el cambio de pauta se apreció principalmente en las colonias urbanas: La Moderna, Alemana y Francesa, ubicadas al suroriente de la ciudad y cercanas a las vías ferroviarias.

“En 1862, los manantiales de San Andrés y San Ramón se estudian para llevar las aguas al Hospicio y al barrio de San J uan de Dios y en 1889 y 1890 las filtraciones del Colli, vía las galerías...sin embargo... la consideración (de) que lo más viable seguían siendo las aguas subterráneas del poniente..." ${ }^{14}$

En las dos últimas décadas del siglo XIX, los gobiernos porfiristas introdujeron infraestructura hidráulica acorde a los ideales industriales que se perseguían, con la idea de que harían más eficiente la accesibilidad de agua en la ciudad.

A la par de la preocupación por la infraestructura hidráulica, la ciudad de Guadalajara experimentó el fenómeno de conglomeración urbana a través de la conexión de caminos entre las localidades de los municipios de Zapopan, Tlaquepaque y Tonalá, aumentando el área de acción para los aguadores.

A finales del siglo XIX, los ayuntamientos regularon de forma institucional la actividad de los arrieros. Anteriormente, era la delegación de policía o comisaría la encargada de observar las pautas de conducta de los arrieros, que oficiaban el servicio de aguadores, y a los representantes de cada grupo les correspondía la organización, limpieza, distribución del área de trabajo y la adhesión de nuevos miembros. Posteriormente, el ayuntamiento se adj udicó dichas facultades.

En el caso de los aguadores, estos tenían que proveer de agua a la capital jalisciense. Además debían de apoyar a los bomberos cuando ocurría algún incendio ${ }^{15}$, ofrecían otros servicios como el de mensajería debido al conocimiento de las rutas de cada uno de ellos a través del radio que les competía y según la autorización del gremio.

En lo concerniente a la infraestructura hidráulica, en 1885 Francisco Tolentino, gobernador de Jalisco, compró en Estados Unidos la primera bomba de vapor para surtir de agua a la ciudad. Emplazó la primera tubería de fierro y colocó 55 hidrantes de palanca en diferentes puntos para que la población se abasteciera.

Además, compró los manantiales y terrenos del balneario Agua Azul, propiedad - en ese entonces-de Antonio Álvarez del Castillo, con la finalidad de obtener una mayor captación de agua con tecnología del momento ${ }^{16}$. Por un lado se esperaba que la

14 Torres Rodriguez, 2013a

15 AHCG, 2016

16 Arana Cervantes, 1980, 27-29. 
Imagen 1. Aguador con carga de ollas en carretilla

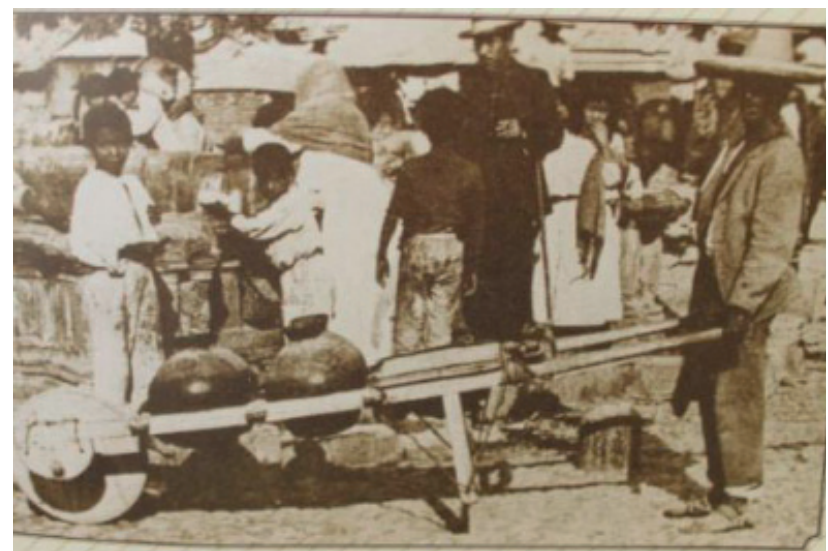

Fuente: Winfield Scott, Guadalajara, Jalisco, México, North America-México-J alisco-Guadalajara, ca. 1908. http:// mediateca. inah. gob. mx

autoridad posibilitara el dinamismo de la dotación de agua y por otro que los comercios, familias de prestigio y el resto de la población pudieran acceder de forma inmediata al recurso hídrico.

Cabe resaltar que la preocupación por tener certeza de más fuentes hídricas accesibles, de aplicar criterios de manejo y tecnologías de infraestructura hídrica para garantizar el recurso de calidad a la población tapatía es sustentada en la escasez aparente y la continua búsqueda de nuevas fuentes de abasto.

En 1893, bajo el gobierno de Luis C. Curiel, se aprobó otro proyecto que pretendía introducir aguas provenientes de los linderos del Cerro del Colli, dicho proyecto tenía contemplada la ampliación de las galerías que Buzeta había diseñado. Los trabajos estuvieron a cargo del ingeniero Gabriel Castaños; no obstante, después de dos años de haberse realizado, el proyecto no había dado el resultado esperado.

Desde entonces se inauguró la práctica de proyectar y construir obras que no se culminarían o que serían rebasadas por los costos, propiciando un gasto público infructuoso hasta entrado el siglo XXI con macroproyectos como la Presa de Arcediano, entre otros, que contaron con presupuesto del erario.

Retomando las obras de finales del siglo XIX, el sistema de abastecimiento era de 430.000 litros de agua cada 24 horas, volumen insuficiente para una población de 80.000 habitantes, debiéndose recurrir a realizar obras de extracción de agua en el cerro del Colli. La ciudad disponía de 6,2 litros por segundo, después del proyecto realizado en dicha área se incrementó la cantidad a 7,84 litros por segundo.

El aumento de un litro era aún insuficiente, por lo que el gobernador decidió la suspensión de las obras que se habían proyectado para una segunda fase en el Colli y propuso que solo se enfocara el esfuerzo técnico a la captación de agua de otras fuentes, pues a fin de cuentas los aguadores facilitaban el acceso de agua potable a los tapatíos.

El programa de obras de agua potable del gobernador Curiel también contemplaba el aprovechamiento de aguas de los acuíferos situados en el bosque "Los Colomos", al norponiente de la ciudad. Al manifestarse el interés de aprovechar los manantiales de algunos terrenos, aparecieron personas que reclamaron la propiedad de los predios. El gobernador, a través del presupuesto público, los adquirió con el fin de evitar demoras.

Para el aprovechamiento de esas aguas, se consideró la utilización de las galerías de Buzeta: "... la producción de todos los manantiales fue concentrada en dos tanques construidos ex profeso, para de ahí bombearla a un punto elevado conocido como El Vigía... el agua tendría que ser mandada por gravedad hacia la ciudad..." ${ }^{17}$

En cuanto al aguador, también se establecieron criterios para reglamentar el desempeño del oficio. Una de las primeras regulaciones fue la inscripción a un censo público de prestadores de servicios. Un reglamento expedido por el Ayuntamiento de la ciudad en 1888 obligaba a todos los prestadores de servicios domésticos, así como a los trabajadores ambulantes entre los que destacan los aguadores, a registrarse con una fotografía en la oficina municipal.

Esta medida hizo que cocineras, cocheros, mozos, niñeras, recamareras, caballerangos, jardineros, porteros, boleros (lustrabotas), peluqueros, vendedores de lotería, sastres y un largo etcétera se volcaran en la docena de establecimientos fotográficos de la ciudad. Con esta disposición de corte decimonónico la fotografía prácticamente estaba al alcance de todas las clases sociales y los patronos ejercían control sobre sus sirvientes pues se quedaban con su carnet durante el tiempo que estaban a su servicio ${ }^{18}$.

\section{LA ÉPOCA POSTREVOLUCIONARIA: LA HIDRÁULICA Y LA TRANSFORMACIÓN DEL OFICIO DE AGUADOR}

Debido a la inestabilidad que la Revolución provocó entre 1909-1917, no se realizaron obras de mejoramiento durante este periodo, las revueltas y la inestabilidad así como la movilización distrajeron la atención de las autoridades. A pesar de dicha circunstancia, las obras realizadas por el gobernador Francisco Tolentino seguían vigentes.

Tras la Revolución Mexicana se instaura el manejo público del agua de forma institucional, en el artículo 27 de la Constitución mexicana de 1917. En tal premisa se establece que la propiedad del agua es de carácter nacional, estipulándose la competencia del gobierno federal para intervenir en el manejo del líquido en cuanto a su destino.

Sin embargo, como menciona Tortolero ${ }^{19}$, el carácter de propiedad nacional sirvió para ejecutar dos acciones: la primera consistió en repartir el agua al mismo tiempo que se realizaba el reparto agrario para satisfacer las demandas de los grupos campesinos; y la segunda, en la creación de grandes obras de aprovechamiento: "La novedad revolucionaria fue, entonces, la utilización del recurso como elemento de política social y de desarrollo económico. Esta situación se mantendría hasta 1983, momento en el cual la injerencia estatal comenzó a verse con recelo..." ${ }^{20}$

\footnotetext{
17 Ibidem, 46.

18 Camacho, 2010, 175.

19 Tortolero Villaseñor, 2000.

20 Ibidem, 100.
} 
La estabilización "post-revolucionaria" incluyó el tema del agua a partir de la estructuración institucional de los organismos que se encargarían de los asuntos hidráulicos. Empieza entonces un proceso de homologación de los criterios que se llevarían a cabo para el manejo del agua en todo el país, ya que en 1926 se creó el primer organismo encargado de los asuntos del agua: la Comisión Nacional de Irrigación (CNI), mediante la Ley de Irrigación con aguas nacionales.

Los objetivos de la ley se enfocaron principalmente en el desarrollo agrario del país. En 1946 dicho organismo se transformó en la primera Secretaría de Recursos Hidráulicos en la que se concentraron todas las funciones del Estado en cuanto al manejo, distribución y construcción de proyectos hidráulicos. Además se realizaron obras de gran infraestructura hidráulica destinada al riego de los campos de cultivo debido a la preocupación alimentaria.

La Secretaría conformó Comisiones Ejecutivas de Cuenca al estilo del Tennesee Valley Authority, de Estados Unidos de América, con el fin de adoptar una regionalización y desarrollar económicamente el país a través de grandes programas hidráulicos como en el sistema norteamericano.

Habría que visualizar al gobierno de ese entonces, que centralizaba todas las actividades de carácter público en su seno, con el énfasis de la regionalización por cuenca adoptada del plan norteamericano y entender los motivos que impulsaron a dicha regionalización.

"En el origen de este poder centralizador estaba la decisión del presidente Plutarco Elías Calles, quien, con su tradición norteña, estaba convencido de que el agua podía servir para fraccionar los latifundios, para impulsar el florecimiento de la clase media agrícola, para modernizar la agricultura y aumentar la producción y para fortalecer la soberanía nacional en el norte del país [región que se caracteriza por su escasez]" 21 .

En la administración del gobernador del estado de Jalisco Sebastián Allende Rojas (1934) se reanudó el interés por mejorar el sistema de abastecimiento de agua para la ciudad. Para tal efecto, ordenó la construcción de la "casa de máquinas" de los manantiales de Agua Azul y ordenó la realización de obras hidráulicas que permitieran aumentar el caudal disponible en el corto plazo.

Guadalajara experimenta un desarrollo económico y cultural en los periodos posteriores a la Revolución mexicana, desarrollo paralelo a la preocupación por abastecerse de agua a largo plazo hasta el punto que se debió replantear los criterios de expansión radial de la urbe.

Cuando la ciudad experimentaba estiaje en las fuentes próximas, el agua adquiría un costo económico mayor y era el momento del dinamismo de los aguadores, quienes transportaban el recurso en vasijas de barro procedentes de Tonalá. Recurrían tanto a las fuentes públicas, arroyos y ríos como a los sifones urbanos, manantiales y ríos en una carrera radial delimitada por el gremio y las autoridades. El agua más accesible provenía principalmente de los manantiales de Agua Azul, al suroriente de Gua-

21 Ibidem, 101. dalajara, de Colomitos al poniente, y sorprendentemente de los arroyos de Tonalá, Tlaquepaque y del río Santiago.

Según el padrón de oficios de Guadalajara dichos aguadores además de acercar agua procedente del río Santiago, aprovechaban los temporales de Iluvia para usar el agua de los arroyos del Rosario y de Enmedio situados a $20 \mathrm{~km}$ de distancia del centro de la ciudad, en la circunscripción del municipio de Tonalá.

Entre las actividades laborales colaterales permitidas por los ayuntamientos municipales cabe destacar que los aguadores se desempeñaban como mensajeros e informantes, o auxiliaban en incendios, al igual que en otras ciudades de México. Los reglamentos eran estrictos en cuanto a las vestimentas y la higiene pues la idea de agua potable también tenía sus implicaciones para el portador.

Con todo, la situación cambiaría con los acontecimientos mundiales de ese tiempo: la II Guerra Mundial y la caída de los precios del petróleo en 1942, que tuvieron serias repercusiones en México. Los proyectos de mejora del servicio público de abastecimiento se suspendieron, por lo que no fue posible dar mantenimiento a las obras que el gobernador Curiel había ordenado construir en el siglo XIX para dotar de agua a fuentes públicas y a la red de surtido domiciliar.

Imagen 2. Aguador con carreta

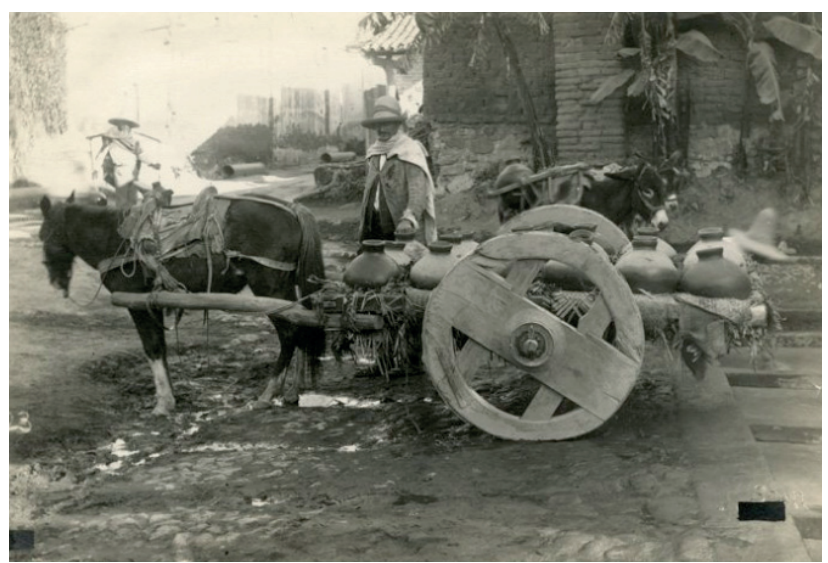

Fuente: INAH, colección Waite/W. Scott. Ca. 1906. http:// mediateca.inah. gob. $\mathrm{mx}$ / islandora_ 74 / islandora

De la misma forma, la carencia de dinero público fue otra circunstancia que agravó la situación durante el periodo de estiaje. A pesar del dinamismo de los aguadores, el aumento del precio por el traslado se convirtió en una limitante para adquirir el recurso hídrico para un segmento de la población.

Antes de la década de los treinta el país se caracterizaba por una baja densidad demográfica y por su atraso industrial. Es decir, México era un país rural con una población distribuida mayoritariamente en el campo. No obstante, la situación mundial posterior obligó a que el país entrara en la dinámica de la industrialización.

"Una vez terminada la fase armada de la revolución, dio comienzo el periodo de reconstrucción del país, con reformas institucionales y políticas cuyo propósito era la consolidación de los primeros 


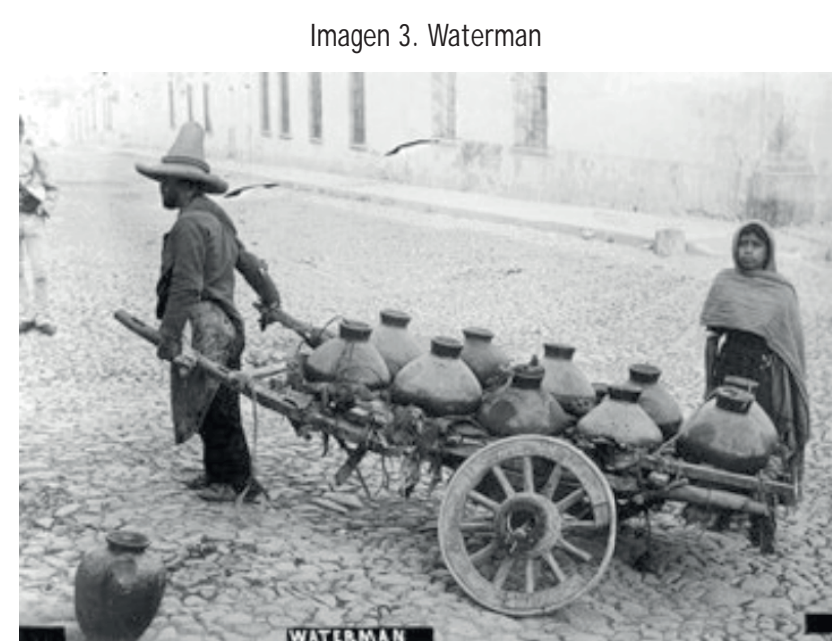

Fuente: INAH, colección Waite/W. Scott. Ca. 1920. http:// mediateca.inah.gob. $\mathrm{mx} /$ islandora_74/ islandora

gobiernos revolucionarios. El crecimiento poblacional durante el decenio siguiente retomó la dinámica que se venía experimentando a principios de siglo: la tasa de incremento demográfico de 1921 a 1930 se elevó a 1,7\% anual, con una población de 16,5 millones de habitantes en el último año" 22 .

Entre 1935 y 1965 se vivió el mayor auge en el aprovechamiento hidráulico para el campo. No obstante, el problema de abastecimiento hídrico se agravó en las ciudades, ya que no se contempló una política efectiva para el aprovechamiento de agua en las ciudades desde el ámbito federal, por lo que cada ciudad comenzó a planificar estrategias para abastecer las actividades urbanas.

En el año de 1943, ante esos problemas, se creó un Consejo de Colaboración Municipal entre Guadalajara, Zapopan y Tlaquepaque, con el objeto de coordinar esfuerzos entre la iniciativa privada y las autoridades de los otros municipios que poseían zona urbana contigua a Guadalajara. La instauración del Consejo de Colaboración y la consecución de zona urbana intermunicipal devino en la instauración de la Zona metropolitana.

Para 1947 la cantidad de agua en la ciudad por habitante aumentó a 133 litros por día. Esto fue posible por la aportación de diferentes fuentes: Colomos al poniente, Agua Azul al sur oriente y los pozos de San Rafael al oriente, del Rastro al sur, de la Colonia Moderna en el centro de la ciudad y de San Rafael-San Andrés al nororiente.

Se aprecia claramente el criterio radial de explotación hídrica. Si el déficit se presentaba siempre había posibilidad de recurrir a los servicios de los aguadores. No obstante, en la medida que los proyectos y las obras hidráulicas proliferaban en la ciudad -como los sifones, fuentes públicas y la extensión de la red de tubería hídrica - el oficio del aguador perdía dinamismo, dinamismo que solo se recuperaba en tiempos de estiaje: pese a que logró rebasar las primeras tres décadas del siglo XX en barrios de la ciudad como Analco, San Carlos, Mexicaltzingo o Mexquitán, con las mismas pautas de acción propias del oficio de este prestador de servicios.

22 Cabrera Acevedo, 1994, 349.
En el mismo año de 1947 se realizaron los primeros estudios para aprovechar las aguas de los ríos Calderón y Verde. Lamentablemente se desechó la opción y se optó por el río Santiago. Luego, en el siglo XXI -ante el estrés hídrico experimentado-se volvió a retomar dichas escorrentías para incorporarlas a proyectos de abastecimiento que incluían el abasto de agua para otras ciudades además de Guadalaj ara.

\begin{abstract}
"Desde la década de 1950 a la actualidad, el interés se ha centrado, fundamentalmente, en atender las demandas de un crecimiento urbano industrial. Para ello los recursos del país en general, entre los que destacan los hidrológicos, han sido orientados para apoyar el crecimiento de una sociedad urbano industrial, así como el de una agricultura dependiente de la industria" ${ }^{23}$.
\end{abstract}

El crecimiento demográfico y económico origina que las ciudades crezcan de manera inusitada, de tal suerte que resulta difícil para los gobiernos poder atender las demandas de una población en crecimiento continuo con necesidades complejas y en diversificación constante. Los investigadores de la política califican a esta situación como de ingobernabilidad, refiriéndose a la falta de capacidad de respuesta del gobierno 0 a la atención ineficiente ${ }^{24}$.

A mediados del siglo XX, la población se había incrementado, la situación general del país dependía de los acontecimientos mundiales y como consecuencia, la situación de las localidades estaba supeditada a las disposiciones del poder del Estado. En el caso de Guadalajara, se tuvo que recurrir a la colaboración del sector público con el privado para poder realizar obras públicas, en especial las de carácter hidráulico. En aquel entonces se realizaron sucesivos estudios para determinar una fuente de abastecimiento duradera y efectiva en el tiempo: el lago de Chapala se convirtió en esa fuente.

Dicha colaboración se debió a la dinámica económica percibida en el país. En la década de los cincuenta en México se vivió un auge industrial que repercutió en el bienestar social, principalmente en las ciudades. Esta situación aceleró el crecimiento de la población y su concentración en las principales ciudades. Al paso del tiempo, las necesidades de agua en la población urbana crecieron de forma acelerada, provocando que los gobiernos no pudieran solventar las necesidades al mismo ritmo.

En el caso de Jalisco y en específico en su ciudad capital, Guadalajara, la administración de Jesús González Gallo, se caracterizó por la reestructuración a fondo del sistema de abastecimiento. Entre 1947 y 1953 se completó la renovación del sistema hidráulico y se recurrió a la perforación de pozos en Tesistán al norte de la ciudad, en territorio zapopano, previa autorización de la Secretaría de Recursos Hidráulicos. Asimismo, surgió un órgano descentralizado encargado de captar, potabilizar y distribuir el agua. El 29 de noviembre de 1952 nació el patronato de los Servicios de Agua y Alcantarillado de Guadalajara.

En el gobierno de Agustín Yánez se consideró que las aguas provenientes del río Santiago serían las adecuadas para solventar la demanda del líquido en la ciudad ante una población en

23 Durán J uárez y Torres Rodríguez, 2002, 497.

24 Borja, 1998. 
Tabla 1. Crecimiento poblacional de J alisco en el siglo XX

\begin{tabular}{|c|c|c|}
\hline Año & Población & $\begin{array}{c}\text { Crecimiento por década } \\
\text { por habitantes }\end{array}$ \\
\hline 1900 & 1.153 .891 & No disponible \\
1910 & 1.208 .855 & 54.964 \\
1921 & 1.191 .957 & -16.898 \\
1930 & 1.255 .346 & 63.389 \\
1940 & 1.418 .310 & 162.964 \\
1950 & 1.746 .777 & 328.467 \\
1960 & 2.443 .261 & 666.484 \\
1970 & 3.296 .586 & 853,325 \\
1980 & 4.371 .998 & 1.075 .412 \\
1990 & 5.302 .689 & 930.691 \\
2000 & 6.321 .278 & 1.018 .589 \\
\hline
\end{tabular}

Fuente: Pérez Peña y Torres, 2001, 7.

incremento atraída por la cuestión urbana. "La ciudad y la zona de influencia son los recipientes de las aguas pluviales que escurren por la cuenca de 46 kilómetros cuadrados hasta la laguna de Chapala y de otros tres kilómetros cuadrados de la cuenca del alto Santiago" 25 .

La ciudad comenzó a recibir agua del lago de Chapala en octubre de 1956. Las obras de captación, conducción, potabilización y distribución del agua procedente del embalse han permitido que fuera la principal fuente de abasto. A pesar de los proyectos gestionados en la década de los noventa, todos llevaron consigo la idea de extracción de dicho cuerpo lacustre.

El crecimiento poblacional de Jalisco se intensificó en la segunda mitad del siglo, presentando más del sesenta por ciento de la población en la ciudad capital de Guadalajara, según se aprecia en la tabla anterior. A partir de la década de los cincuenta el porcentaje de crecimiento casi se duplicó pasando del 12,9\% de crecimiento en la década de los cuarenta a los 23,1 \% en la década de los cincuenta, triplicándose en la década de los sesenta. En esta última década el crecimiento empezó a ser regulado mediante campañas de concienciación y de control de la natalidad. A pesar del esfuerzo, el crecimiento natural contribuyó al incremento de la población del estado.

Como se observa en la tabla 2, de 1950 a 2000 la población de la zona metropolitana se incrementó siete veces más debido a la conurbación de la ciudad a municipios aledaños. De tal suerte que la atención del gobierno estatal al tema hídrico se centró en buscar fuentes alternativas y duraderas para abastecer de

\footnotetext{
25 Arana Cervantes, 58-59.
}

agua a la zona metropolitana a la par que se implementan proyectos para el saneamiento de aguas.

La ciudad de Guadalajara experimentó un proceso de crecimiento poblacional y territorial, característico del fenómeno de conurbación de la ciudad hacia otros municipios ${ }^{26}$, sin que este se detuviera hasta que las obstrucciones fisiográficas naturales frenaran la "expansión de la ciudad".

Respecto al agua, los gobiernos se han preocupado más en solventar la escasez que en buscar soluciones a la integración de los espacios urbanos.

"La configuración y el funcionamiento del sistema urbano de la ciudad de Guadalajara rebasaron la capacidad de oferta de las aguas subterráneas del acuífero Tesistán; la dimensión que fue adquiriendo esta metrópoli exigió que se solucionara una serie de problemas de abastecimiento, los cuales se fueron resolviendo desde una óptica parcial, que permitió paliar a corto plazo las necesidades de la creciente frontera urbana" 27.

Durante la década de los setenta $-y$ a finales de la de los ochenta del siglo XX- se modificaron las estructuras institucionales así como las legales; la Secretaría de Recursos Hidráulicos cedió el lugar a la Secretaría de Agricultura y Recursos Hidráulicos (SARH), tomando en cuenta que la cuestión agraria era inseparable de la hidráulica.

En 1975 se elaboró el primer Plan Nacional Hidráulico (PNH) con el fin de programar y planificar acciones públicas y se instituyó la Comisión del Plan Nacional Hidráulico (CPNH) destinada

Tabla 2. Evolución de la población en la zona metropolitana y en el municipio de Guadalajara a partir de 1950

\begin{tabular}{|c|c|c|c|l|}
\hline Año & $\begin{array}{c}\text { Zona metropolitana } \\
\text { de Guadalajara }\end{array}$ & $\begin{array}{c}\text { Municipio } \\
\text { de Guadalajara }\end{array}$ & $\begin{array}{c}\text { Promedio de Extracción } \\
\text { de agua de Chapala } \\
\mathrm{m}^{3} / \mathrm{seg}\end{array}$ & $\begin{array}{l}\text { Fuentes secundarias } \\
\text { de extracción de agua. } \\
\text { Toponimia (municipio) }\end{array}$ \\
\hline 1950 & 484.532 & 381.138 & 5,96 & $\begin{array}{l}\text { Pozos de Tesistán } \\
\text { (Zapopan) }\end{array}$ \\
\hline 1960 & 907.421 & 740.304 & 5,96 & \\
\hline 1970 & 1.533 .485 & 1.199 .391 & 4 & $\begin{array}{l}\text { Tesistán (Zapopan) } \\
\text { Los Colomos } \\
\text { (Guadalajara) } \\
\text { Agua Azul (Guadalajara) } \\
\text { El Deán (Guadalajara) } \\
\text { y pozos diversos de } \\
\text { Zapopan }\end{array}$ \\
\hline 1980 & 2.322 .580 & 1.626 .152 & 4 & $\begin{array}{l}\text { Presa Elías González } \\
\text { Chávez, sistema Tesistán } \\
\text { y sistema Toluquilla }\end{array}$ \\
\hline 1995 & 3.461 .819 & 1.633 .216 & & $\begin{array}{l}\text { Presa Elías González } \\
\text { Chávez, sistema Tesistán } \\
\text { y sistema Toluquilla }\end{array}$ \\
\hline 2000 & 3.689 .792 & 1.647 .720 & 7,5 & $\begin{array}{l}\text { Presa Calderón y pozos } \\
\text { subterráneos, Sistema } \\
\text { Tesistán y sistema } \\
\text { Toluquilla }\end{array}$ \\
\hline
\end{tabular}

Fuente: Elaboración propia. Datos extraídos de Pérez Peña y Torres, 2001, 8.

26 Rojas Ramírez, 2013.

27 Aguirre Jiménez, 2004, 25. 
a sistematizar los procesos de planificación del sector agua. En 1981 se crea un nuevo programa hidráulico como parte del Plan Global de Desarrollo. No obstante, en esta década el país sufrió una crisis que obligó a reconfigurar las estrategias correspondientes al sector agua: "Las restricciones e incertidumbres de esta crisis transformaron la planificación en una programación a corto plazo y en una búsqueda continua de soluciones presupuestarias para terminar las obras iniciadas" 28.

Para 1985 el gobierno federal desconcentra las actividades de la SARH vinculadas al agua en la Subsecretaría de Infraestructuras Hidráulicas pues los asuntos hidráulicos rebasaron la capacidad de atención de la secretaría y por ello fue necesario crear una institución que se encargara de los problemas del agua ocasionados por el crecimiento de la población y del gran cambio global.

El CNPH se transforma en Instituto Mexicano de Tecnología del Agua (IMTA) cuyo papel en esta época fue la promoción y la instauración de políticas que permitieran: el uso eficiente del agua; la protección y el control de la calidad del agua; el tratamiento y la reutilización de las aguas negras y la formación de los recursos humanos y el desarrollo tecnológico.

La desconcentración ${ }^{29}$ se trasladó hasta llegar a modificar el artículo constitucional 115: en dicha reforma se le atribuyó al municipio la administración de los servicios urbanos de agua y saneamiento. Sin embargo, la centralidad del poder aún era visible en la práctica ya que la toma de decisiones se realizaba en los altos niveles y la delegación de responsabilidades a los otros ámbitos de gobierno más que beneficiarlos les perjudicó, pues los gobiernos locales no sabían cómo realizar lo estipulado en la reforma del artículo 115: "Al final de los años ochenta, la situación del sector del agua empeoró. Problemas de disponibilidad existen en los dos tercios del país. La eficacia en el uso del agua está muy baja y la calidad de las aguas se deteriora" 30 .

En 1989, bajo el gobierno de Carlos Salinas de Gortari (19891994), se crea la Comisión Nacional del Agua, como un organismo descentralizado de la Secretaría de Agricultura y Recursos Hidráulicos (SARH). El organismo tenía en su cometido tres direcciones básicas: Desarrollo de infraestructuras para satisfacer una demanda creciente, aumento de la eficacia en el uso del agua y priorizar el control y la disminución de la contaminación del agua.

De la misma forma, se propuso y aprobó la Ley de Aguas Nacionales en sustitución de la Ley Federal de Aguas de 1972. El propósito fue crear un marco regulador moderno acorde a la realidad y al discurso político referente a la descentralización. La ley instaura como principios fundamentales, la gestión integrada del agua, la planificación y la programación hidráulica, una mayor participación de los usuarios del agua y la seguridad jurídica en términos de derecho de uso del agua ${ }^{31}$.

28 www. francia. org. mx/ coopcyt/ coopenAgua/ AguaMetLAN. htm

29 En Administración Pública la desconcentración supone la delegación de poderes o responsabilidades a un órgano de la administración pública. https:/ / revistas-colaboracion.juridicas. unam. $\mathrm{mx} /$ index. php/ rev-administracion-publica/ article/ view/ 18461/ 16591.

30 Ministère des Affaires Étrangères, 2006

31 Idem.
En otro orden de ideas, la expansión de la ciudad y el material utilizado para la impermeabilización con malla asfáltica y concreto hidráulico impide que las aguas pluviales penetren al subsuelo provocando que los mantos freáticos no se recuperen al tiempo que se sustrae el agua del subsuelo. Aunado a ello, está el problema de las aguas residuales provenientes de la actividad cotidiana de la sociedad. Estas aguas son desechadas en las cuencas fluviales provocando que sus paisajes se deterioren, en especial en los municipios aledaños al conurbado.

La primera idea que se concibió consistía en que el agua contaminada fuera guiada por el cauce de las cuencas al mar en la idea de que en el transepto, la acción natural de oxigenación por efectos fisicoquímicos de ozonificación natural y arrastre remediaría o mitigaría la situación, sin tener en cuenta la acumulación sedimentaria de contaminantes (sulfatos, fosfatos, nitratos metales pesados, residuos antibióticos y hormonales) aunados a la acumulación creciente de coliformes que provocaron eutrofización y problemas de salud pública.

Ante la problemática de contaminación de los sistemas hídricos surgió la necesidad de sanear las aguas residuales. En la actualidad se está determinando la afectación a la salud pública, el costo de los daños y las posibilidades reales de regeneración tras centenares de años en constante abatimiento y contaminación.

Según el Consejo Estatal de Agua y Saneamiento (CEAS), en el 2004, la ciudad necesitaba $12,5 \mathrm{~m}^{3} /$ seg y solo recibía $9 \mathrm{~m}^{3} / \mathrm{seg}$, lo cual se traduce en un déficit en el uso de agua. En promedio, cada habitante de la ciudad requiere 205 litros para solventar sus actividades (considerando gasto global, habitante, comercio y empresa), dicha cantidad supera la media internacional de 100 litros por día. Habría que preguntarse si existen otras ciudades igualmente pobladas en las que la ración de abastecimiento sea parecida 0 inferior a la que Guadalajara recibe. El problema de abastecimiento en la zona conurbada es mas bien un problema de administración del recurso -tanto institucional como domésticoque de escasez del recurso, a pesar de la política nacional.

Respecto al oficio de aguador, a partir de la década de los ochenta experimentó una serie de transformaciones en la ciudad de Guadalajara ya que ante las reformas estructurales y el aceleramiento en la introducción de infraestructura hidráulica en la ciudad, el oficio tradicional quedó en desuso desde la perspectiva de organización y propiedad de los insumos de trabaj $0^{32}$.

El oficio de aguador desapareció de los archivos referentes a permisos de Oficios y Profesiones en el Ayuntamiento de Guadalajara y dio paso al empleado repartidor de agua, cuya persona es contratada por las empresas potabilizadoras para distribuir el recurso a través de rutas de comercialización previamente establecidas por sus equipos de mercadeo.

La actividad de comercializar el agua potable adquirió un enfoque propio de la dinámica neoliberal, en el que la libertad de mercado responde a las leyes de oferta y demanda, sin que el Estado intervenga más que para regular que el origen de lo comercializado y su uso sean lícitos.

32 Pacheco-Vega, 2015. 
El éxito del nuevo esquema de comercialización del agua en Guadalajara se debe, en primera instancia, a las sospechas sociales sobre la inocuidad del recurso proveído por el organismo operador SIAPA. Ante la percepción social, algunos empresarios locales detectaron la oportunidad de invertir en la distribución comercial de agua purificada y embotellada. En segunda instancia, la comercialización del agua a través del esquema de envasado se ofrece, mercadológicamente, aludiendo a ideas preconcebidas sobre la importancia del "envasado" y sus efectos benéficos sobre la salud.

Cabe mencionar que, a finales de la década de los setenta, el organismo encargado del Sistema Intermunicipal de Agua Potable y Alcantarillado de la ciudad (SIAPA), experimentó problemas de financiamiento y complicaciones técnicas en el mantenimiento de las redes de distribución de agua y alcantarillado ${ }^{33}$. Estos problemas repercutieron en la percepción social sobre la calidad del agua. Como consecuencia, surgió la oportunidad para nuevos inversores interesados en la comercialización del recurso, a través del diseño de rutas de venta y presentación del "producto" envasado. Desde entonces se consolidó el mercado de agua en la ciudad. Los mercados de agua rompen con la idea de gratuidad y subvención ${ }^{34}$.

Las empresas potabilizadoras utilizan eslóganes referentes a la salud pública relacionados con el sabor, olor y color del agua que ellos ofrecen. El esquema de distribución aplicado al nuevo gremio empresarial utiliza la base usada por los aguadores y sus gremios: la venta a domicilio.

El nuevo esquema del aguador, o desaparición del tradicional, implica la distribución de agua en "garrafones", recipientes de vidrio o PET, en automóviles operados por empleados de las empresas potabilizadoras que trasladan dichos recipientes a los domicilios de los consumidores.

A pesar de la infraestructura hidráulica existente en la ciudad, no se logra solventar el abasto hídrico en el estado de Jalisco, según datos ofrecidos por la Comisión Estatal del Agua. El organismo operador (SIAPA) carece de capacidad financiera para atender las averías existentes en la imbricada red de dotación urbana, de la cual se pierde -a causa de fugas-el $18 \%$ de agua que contabiliza para su distribución ${ }^{35}$.

\section{Conclusiones}

Por consiguiente, el oficio de aguador -aunque diferente y bajo esquemas laborales propios del modelo neoliberal- sigue vigente en los empleados de las actuales transaccional es con concesión de explotación del agua para su comercialización en botellas de PET -garrafones-, cuyo destino es el consumo humano.

El repartidor de agua es un empleado contratado por las empresas potabilizadoras cuya actividad es vender dichos garrafones llenos de agua previamente purificada. Dichas empresas son propietarias de los medios de trabajo usados para el transporte

33 SIAPA, 2012

34 Embid Irujo, 1997, 13-16.

35 SIAPA, 2012 de los envases, camiones en los que movilizan "el producto", dejando al empleado solo con la propiedad de su fuerza de trabajo, a diferencia de los aguadores tradicionales, quiene poseían, en la mayoría de los casos, sus insumos de trabajo.

Dicha situación refleja el cambio del oficio de aguador, quien durante trescientos años dotaba del recurso vital a la población de Guadalajara que requería el agua a causa de su escasez. Actualmente, la comercialización del agua responde a las percepciones sociales sobre la calidad del recurso que dota el organismo operador a diferencia del agua comercializada por las empresas, las cuales tienen que cumplir con parámetros de calidad para poder operar en el mercado del agua.

Cabe destacar que al gunas grandes empresas potabilizadoras - desde finales de la década de los setenta- ubicaron sus naves industriales en las cercanías de los veneros del Agua Azul: de aquel lugar se surte también el organismo operador. En dicha década iniciaron operaciones las empresas potabilizadoras de agua Arcoiris y Pureza, cuyos productos ofrecidos en el mercado eran agua potable y hielo.

Actualmente la competencia en el mercado neoliberal propicia que las grandes empresas transnacionales con ventajas competitivas desplacen a las potabilizadoras pequeñas, como es el caso de agua Bonafont, de grupo Danone S. A. de C. V., cuyo corporativo inició actividades a principios de la primer década del presente siglo y estableció actividades logísticas a escasos doscientos metros de la empresa potabilizadora Arcoiris.

En la actualidad, se incrementaron las funciones de los organismos que manejan el agua; además del abastecimiento se incluyó en la Ley de Aguas Nacionales el saneamiento como función prioritaria. Por otra parte, surgió el interés en los empresarios por incursionar en el mercado, que anteriormente estaba enfocado al oficio de aguador y que bajo la lógica del mercado neoliberal se transforma ahora en una modalidad de distribución de un bien social bajo el esquema de comercializarlo en el mercado.

Las empresas modernas operan bajo la lógica comercial de vender agua potabilizada a través de botellones de PET, entre otros productos, mediante un esquema de distribución en unidad motorizada (camión), operada por dos empleados, repartidores de agua, cuya función es vender los garrafones a domicilio.

Las empresas operan con permisos que les dota el Estado para extraer agua de sus fuentes naturales, como se evidencia en casos específicos como los de Coca-Cola y Nestlé. Posteriormente, están obligados a cumplir con las normas técnicas oficiales (en el caso mexicano se nombran Normas Oficiales Mexicanas) que les obliga a potabilizar el recurso para poder trasladarlo y comercializarlo.

El proceso enunciado refiere a lo que Pacheco-Vega denomina privatización del agua ${ }^{36}$ ya que este es un bien público que no debería estar suj eto a costos que impliquen márgenes de ga-

36 Norma oficial mexicana NOM-127-SSA1-1994: "Salud ambiental, agua para uso y consumo humano. Límites permisibles de calidad y tratamientos a que debe someterse el agua para su potabilización". Fuente: http:// www.salud.gob. mx/ unidades/ cdi/ nom/ 127ssa14. html 
nancia, salvo los asociados con su manejo y saneamiento, que -a su vez- presuponen otras fases en el proceso de gestión. Dichas empresas operan bajo el esquema de comercialización a precios más elevados, ya que además de los costos asociados a la potabilización y distribución se busca un margen de ganancia. A diferencia de la cuota estipulada por los organismos operadores que presupone costos asociados a los servicios de operación técnica y administrativa, potabilización y distribución. Con esto, en teoría, se garantiza el Derecho al agua, ya que se responde a los principios de gratuidad y subvención. Asimismo, en el artículo $4^{\circ}$ párrafo sexto de la Constitución de los Estados Unidos Mexicanos, se establece que "Toda persona tiene derecho al acceso, disposición y saneamiento de agua para consumo personal y doméstico en forma suficiente, salubre, aceptable y asequible".

Las empresas potabilizadoras buscan un fin lucrativo, para alcanzarlo compiten entre sí a través de campañas publicitarias $y$, en la medida de lo posible, cumpliendo con las obligaciones legales exigidas. Lo que les facultó para comercializar los recursos hídricos, fueron los permisos para el aprovechamiento del recurso y el cumplimiento de los parámetros de salubridad de la Norma Oficial Mexicana Nom-127-SSA1-1994.

En lo referente a la comercialización de un bien público, cabe preguntarse si las empresas -al obtener un lucro por la mercantilización del agua- están incurriendo en un acto de ilegalidad. 0 es acaso que el objetivo principal de sus operaciones comerciales con el recurso sea ofrecer altos estándares de calidad impuestos a un recurso natural, que deviene como bien social ${ }^{37}$, acorde a la capacidad de pago de cada consumidor?

Si bien el esquema de comercializar el recurso hídrico restringe el acceso al agua potable según la capacidad de pago, el Estado -a través de sus instituciones enfocadas al manejo- debería garantizar el acceso al agua de calidad.

En el caso de Guadalajara, la red de infraestructura hidráulica del Sistema Intermunicipal de Guadalajara es obsoleta, por lo que el agua potable tiene que comprarse a las potabilizadoras. Como se ha venido argumentando, la percepción social de la calidad del agua aún prevalece en los tapatíos.

Uno de los principales problemas que los gobiernos deben solucionar es la escasez de agua de calidad a través de la vinculación de los sectores económicos, académico-científicos y sociales, como una tríada paralela al desarrollo sustentable.

\section{Bibliografía}

Aguirre Jiménez, Alma A. 2004: "La construcción de represas para abastecimiento de agua potable en zonas urbanas y su efecto en la sostenibilidad ambiental. El caso de la zona metropolitana de Guadalajara", en Boletín del Archivo Histórico del Agua, 28, 24-32.

Arana Cervantes, M. 1980: Agua para todos. La lucha de una ciudad por apagar su sed. Guadalajara, Gobierno del Estado de J alisco-SIAPA.
Ávila, P. y González García, A. R. 2012: "Agua para las ciudades en el Porfiriato. El caso de Guadalajara, México", en Revista del Colegio de San Luis, 2, 4, San Luis Potosí, 10-34.

Borja, R. 1998: "Gobernabilidad", en Enciclopedia de la política. MéxiCO, FCE.

Camacho Becerra, Juan A. 2010: "Arte y simulación: imágenes de las clases populares en Guadalajara durante el Porfiriato", en Letras Históricas, 2, Guadalajara, 157-185.

Cabrera Acevedo, G.1994: "El estado mexicano y las políticas de población", en Alba, F. y Cabrera, G. (comps.): La población en el desarrollo contemporáneo en México. México, El Colegio de México.

Durán J uárez, J. M. y Torres R., A. 2002: "Crisis ambiental en el lago de Chapala y el abastecimiento para Guadalajara", en Boehm Schondube, B.; Durán J. . J. M.; Sánchez Rodríguez, M. y Torres Rodríguez, A.: Los estudios del agua en la cuenca Lerma-Chapala-Santiago. Zamora, El Colegio de Michoacán.

Embid Irujo, A. 1997: "Precios y mercados del agua", en Ingeniería del Agua, 4, I, 13-16. DOl: https:// doi.org/ 10.4995/ ia.1997.2711.

López Cotilla, M. 1983: "Fuentes y Acueductos", en Noticias geográficas y estadísticas del departamento de alisco. Guadalajara, Gobierno del Estado de J alisco, 34-35.

Ministère des Affaires Étrangères. 2006: "Cooperación técnica sector agua. 'Marco institucional/ introducción histórica de 1917-1988'", en Panorama del agua. México, Embajada de Francia (en www. francia. org. mx/ coopcyt/ coopenAgua/ AguaMetLAN. htm).

Pacheco-Vega, R. 2015: "Agua embotellada en México: de la privatización del suministro a la mercantilización de los recursos hídricos", en Espiral, 22, 63, 221-263. Recuperado en 11 de mayo de 2017, de http:// www. scielo.org. mx/ scielo. php?script=sci_ arttext\&pid=S1665-05652015000200007\&lng=es\&tIng=es.

Pérez-Peña, 0. y Torres-González, G. 2001: "La insaciable sed de agua de la zona metropolitana de Guadalajara", en Renglones, 49, Tlaquepaque, 5-19.

Roj as Ramírez, J osé J. P. 2013: "Dilemas del desarrollo regional hidráulico al interior de México: conflictos en la gestión regional del agua y nueva fórmula política en la cuenca Lerma-Chapala", en Historelo: Revista de Historia Regional y Local, 5, 9, Bogotá, 318-352. DOl: https:// doi. org/ 10.15446/ historel0.v5n9. 36208.

Sartorius, C. C. (Trad. Mercedes Quijano), 1991: México, paisajes y bosquejos populares: México y los mexicanos. México, Centro de Estudios de Historia de México CONDUMEX.

Shiva, V. 2003: "La abundancia se convierte en escasez", en Las guerras del agua. Privatización, contaminación y lucro. México, Siglo XXI editores, 16-31.

SIAPA, 2012: Presupuesto 2013. Guadalajara, Siapa.

Torres Rodríguez, A. 2013a: "Infraestructura hidráulica en Guadalajara para el abastecimiento de agua potable: el caso de sustentabilidad en las galerías filtrantes de Guadalajara", en Relaciones. Estudios de historia y sociedad, 136, Zamora, 317-357.

Torres-Rodríguez, A. 2013b: "Abastecimiento de agua potable en las ciudades de México: el caso de la zona metropolitana de Guadalajara", en Agua y Territorio, 1, Jaén, 77-90, DOl: https:// dx. doi. org/ 10.17561/ at. v1i1.1035.

Tortolero Villaseñor, A. 2000: "El siglo XX", en El agua y su historia, México y sus desafíos hacia el siglo XXI. México, edit. Siglo XXI, 100-114.

37 Shiva, 2003 